Atualização em Reumatologia

UPDATE IN RHEUMATOLOGY

Responsável: Boris Afonso Cruz

\title{
Atualização em Doença de Behçet
}

\section{Update in Behçet's Disease}

\author{
Seleção de artigos e comentários feitos por
}

Boris Afonso Cruz $z^{(1)}$

\begin{abstract}
A doença de Behçet é entendida como uma vasculite sistêmica que envolve vasos de qualquer calibre. Ainda que algumas manifestações sejam características, como a aftose bipolar, não existem alterações laboratorias ou histopatológicas definitivas da doença, sendo o diagnóstico dependente de uma avaliação clínica criteriosa. A doença de Behçet é potencialmente grave, especificamente quando da verificação de uveíte, envolvimento do sistema nervoso central ou de comprometimento inflamatório de grandes vasos, como aortite. O diagnóstico precoce e a instituição de tratamento adequado são determinantes do prognóstico. Publicamos neste número uma revisão da literatura recente sobre aspectos clínicos, fisiopatológicos e terapêuticos da doença de Behçet.
\end{abstract}

Amoura Z, Dode C, Hue S, et al: Association of the R92Q TNFRSF1A mutation and extracranial deep vein thrombosis in patients with Behçet's disease (Associação da mutação do R92Q TNFRSF1A e trombose venosa extracranial em pacientes com doença de Behçet). Arthritis Rheum 52:608-1 1, 2005. Hospital Pitié-Salpetriere, Paris, França.

A fisiopatologia da doença de Behçet envolve o TNF. Os autores avaliaram a mutação R92Q do receptor TNF superfamília 1A (R92Q TNF 1A) em pacientes europeus e dos países norte-africanos Marrocos, Tunísia e Argélia. Foram estudados 112 pacientes (74 europeus e 38 norteafricanos), comparados a 909 controles europeus e 89 controles da composição dos países Marrocos, Tunísia e Argélia. A freqüência da mutação R92Q TNF 1A foi 5/74 (6,8\%) pacientes europeus e 12/909 (1,3\%) controles europeus $(P=0,006)$. Em nenhum controle dos países africanos foi encontrada a mutação. Um paciente africano apresentou a mutação. Do ponto de vista clínico, pacientes com mutação R92Q TNF 1A tinham menor freqüência de envolvimento ocular $(16,7$ vs. $62,5 \% ; P=0,27)$ e maior frequiência de trombose venosa periférica (100\% vs. $26,8 \% ; P=0,0002)$. Após a correção de Bonferroni para comparações múltiplas, manteve-se a significância estatística da associação entre a mutação R92Q TNF 1A e trombose periférica. Ainda que a contribuição desta mutação na fisiopatologia da doença não esteja clara, os autores entendem que este novo achado pode ajudar a entender o estado pró-trombótico complexo que os pacientes com doença de Behçet apresentam.

Sonoda KH, Inaba S, Ariyama A, Kawano YI, Saniabadi A, Ishibashi T: Therapeutic neutrophil apheresis in patients with ocular Behçet's disease (Aférese terapêutica de neutrófilos em pacientes com doença de Behçet ocular). Arch Ophthalmol 123: 267-9, 2005. Department of Ophthalmology, Graduate School of Medical Sciences, Kyushu University, Fukuoka, Japão.

Os autores avaliaram a resposta terapêutica da aférese de neutrófilos em quatro pacientes com doença de Behçet e envolvimento ocular grave. Os pacientes foram inicial- mente acompanhados por seis meses, então submetidos a cinco sessões da aférese e seguidos por outros seis meses. A média do número de recidivas da inflamação ocular foi

1. Serviço de Reumatologia do Biocor Instituto, Nova Lima, MG, Brasil. 
menor nos seis meses após a aférese $(2,5 \pm 0,6$ vs $5,0 \pm 1,8$; $P=0,04)$ e três quartos dos pacientes não apresentou ataques maiores. Todos os pacientes apresentaram alguma melhora da acuidade visual. Mesmo não tendo sido o estudo realizado para avaliar comprometimento extra-ocular, foi verificado melhora da diarréia e de úlceras orais em dois pacientes. Não houve efeito adverso no período de seguimento.

Tugal-Tutkun I, Onal S, Altan-Yaycioglu R, Huseyin Altunbas H, Urgancioglu M: Uveitis in Behçet's disease: an analysis of 880 patients (Uveíte em doença de Behçet: uma análise de 880 pacientes). Am J Ophthalmol 138: 373-80, 2004. Department of Ophthalmology, Istanbul University, Istanbul Faculty of Medicine, Istambul, Turquia.

Em um estudo retrospectivo, os autores descrevem características demográficas e clínicas de 880 pacientes com doença de Behçet e envolvimento ocular, acompanhados na instituição entre 1980 e 1998. Sessenta e oito por cento dos pacientes eram homens e o comprometimento ocular foi bilateral em 78,1\% dos casos. A idade média do início da uveíte foi 28,5 anos nos homens e 30 anos nas mulheres. Lesões do pólo posterior e por conseqüência complicações ameaçadoras à visão foram mais freqüentes nos homens. Análise por curva de sobrevida mostrou que o risco de perda da visão em 5 e 10 anos de seguimento foi maior nos homens (21\% e 30\%, respectivamente) em comparação às mulheres (10\% e $17 \%$, respectivamente). Pacientes do sexo masculino que iniciaram seguimento a partir de 1990 apresentaram menor risco de perda da visão em comparação com pacientes que iniciaram seu acompanhamento ainda na década de 80 .

Ohno S, Nakamura S, Hori S, Ohno S: Anti-Tumor Necrosis Factor Alpha Antibody in the Treatment of Behçet's Disease (Anticorpo anti-TNF no tratamento de doença de Behçet). Inter Ophtalmol Clin 45: 179-89, 2005. Instituição: Department of Ophthalmology and Visual Sciences, Hokkaido University Graduate School of Medicine, Sapporo, Japão.

Os autores realizaram um estudo aberto e prospectivo para avaliar a eficácia de infliximabe em pacientes com uveíte associada à doença de Behçet. Os pacientes deveriam apresentar ao menos um episódio de inflamação ocular em um período de 14 semanas, avaliado retrospectivamente antes do início do estudo. Os pacientes foram observados prospectivamente por mais 14 semanas. O número de episódios inflamatórios oculares e a acuidade visual foram aferidos neste período de observação e por 14 semanas de seguimento após o início do tratamento. Treze pacientes com panuveíte e vasculite retiniana atribuída à doença de Behçet foram incluídos no estudo e receberam infusões de infliximabe nas doses de $5 \mathrm{mg} / \mathrm{kg}(\mathrm{n}=7)$ e $10 \mathrm{mg} / \mathrm{kg}(\mathrm{n}=$ 6) nas semanas $0,2,4,6$ a 10. O número de episódios de inflamação ocular foi menor no período de seguimento em comparação ao período de observação antes do tratamento no grupo que recebeu a dose de $5 \mathrm{mg} / \mathrm{kg}(1,0 \pm$ 2.2 vs $4,0 \pm 2.2 ; P=0,031)$ e no grupo que recebeu a dose de $10 \mathrm{mg} / \mathrm{kg}(0,2 \pm 0,4$ vs $3,8 \pm 1,9 ; \mathrm{P}=0,031)$. A diferença entre os dois grupos não foi significativa $(P=0,617)$. A acuidade visual melhorou em $5 / 7$ pacientes do grupo que recebeu dose de $5 \mathrm{mg} / \mathrm{kg}$ e $4 / 6$ do grupo que recebeu $10 \mathrm{mg} / \mathrm{kg}$. No período de observação, efeitos adversos caracterizados como infecciosos ocorreram em cinco pacientes do grupo de $5 \mathrm{mg} / \mathrm{kg}$ e dois do grupo de $10 \mathrm{mg} / \mathrm{kg}$. Efeitos adversos atribuídos à infusão ocorreram em cinco pacientes de cada grupo, não tendo sido necessária a suspensão do tratamento. Um evento adverso considerado como grave - tuberculose miliar com envolvimento meníngeo e respiratório - ocorreu em um paciente do grupo de $10 \mathrm{mg} / \mathrm{kg}$, tendo sido suspenso o tratamento a partir da segunda infusão. Os autores entendem que infliximabe pode ser uma alternativa terapêutica em pacientes com doença de Behçet e uveíte refratária ao tratamento combinando com corticóide e imunossupressores como ciclosporina. 
Melikoglu M, Fresko I, Mat C, et al: Short-term trial of etanercept in Behçet's disease: a double blind, placebo controlled study (Ensaio clínico curto de etanercepte em doença de Behçet: um estudo duplo cego controlado com placebo). J Rheumatol 32: 98-105, 2005. The Behçet's Syndrome Research Center, Cerrahpasa Medical Faculty, Istambul, Turquia.

O objetivo do estudo foi avaliar a eficácia de etanercepte no teste da patergia, prova do monourato de sódio e em sintomas cutâneos e articulares de doença de Behçet. Quarenta pacientes do sexo masculino com teste da patergia e prova do monourato de sódio positivos foram randomizados para receber etanercepte $25 \mathrm{mg}$ por via SC 2 vezes por semana $(n=20)$ e placebo $(n=20)$ por 4 semanas. Após o período de observação, não houve diferença nas medidas do teste da patergia e do monourato de sódio entre os grupos. O número médio de úlceras, lesões nodulares e papulopustulares foi menor no grupo que recebeu etanercepte. A probabilidade de estar sem úlceras orais ou lesões nodulares foi maior no grupo que recebeu o tratamento ativo $(P=0,0017$ e $P 0,0002$, respectivamente). Os autores concluem que, ainda que não influencie o teste da patergia ou a prova do monourato de sódio, etanercepte pode ser eficaz contra manifestações muco-cutâneas da doença.

Calamia KT, Schirmer M, Melikoglu M: Major vessel involvement in Behçet's disease (Envolvimento vascular maior em doença de Behçet). Curr Opin Rheumatol 17: 1 8, 2005. Mayo Clinic College of Medicine, Jacksonville, Florida; Innsbruck Medical University, Innsbruck; e University of Istanbul, Cerrahpasa Faculty of Medicine, Istambul, Turquia.

Neste artigo de revisão, os autores discutem os principais aspectos clínicos, fisiopatológicos e terapêuticos da vasculite de vasos de maior calibre na doença de Behçet. Sabe-se que o envolvimento de vasos de maior calibre como aortite, vasculite da circulação pulmonar e eventos trombóticos periféricos e/ou viscerais acontecem em um quarto a um terço dos pacientes, com prevalências semelhantes em diferentes regiões do mundo. Após um primeiro evento vascular, os pacientes apresentam risco aumentado de novos eventos e por isso pior prognóstico. Eventos vasculares maiores são também mais freqüentes em homens e pacientes mais jovens. Este fato é importante pois estes pacientes devem ser triados para novas manifestações de vasculite de vasos de maior calibre e receber tratamento mais agressivo. Existem diferenças no comportamento clínico de tromboses e aneurismas nestes pacientes, em comparação com pacientes com aterosclerose ou outras doenças. A inflamação nas artérias é aguda e destrutiva, causando rapidamente a formação de aneurismas e com maior incidência de ruptura e/ou sangramento. A oclusão venosa é caracterizada pela extensiva formação de trombos aderentes, sem evidência clínica de trombo-embolismo. A trombose venosa pode evoluir a despeito da anticoagulação. Trombos, aneurismas e pseudoaneurismas podem complicar cirurgias e procedimentos invasivos, refletindo um efeito "patergia-like" na parede vascular. A vasculite, quando ativa, pode cursar com repercussão sistêmica e a elevação de provas inflamatórias pode auxiliar no seguimento da atividade da doença. A vasculite de vasos maiores na doença de Behçet e seu processo inflamatório sistêmico requerem seu reconhecimento, o entendimento de suas consequiências e tratamento agressivo, que pode incluir combinação de anticoagulação com heparina, cumarínicos e anti-agregantes plaquetários, associados ou não a corticóide e/ou imunossupressores.

Karacorlu M, Mudun B, Ozdemir H, Karacorlu SA, Burumcek E: Intravitreal triamcinolone acetonide for the treatment of cystoid macular edema secondary to Behçet's disease (Triamcinolona acetonida para tratamento de edema macular cistóide secundário à doença de Behçet). Am J Ophthalmol 138: 289-91, 2004. Istanbul Retina Institute, Istambul, Turquia.

Os autores realizaram um estudo aberto prospectivo para avaliar a eficácia da infiltração intraocular de triamcinolona acetonida em edema macular cistóide secundário à doença de Behçet, refratário ao tratamento clínico. Dez olhos em 
10 pacientes foram submetidos à infiltração com $4 \mathrm{mg}(0,1 \mathrm{ml})$ de triamcinolona acetonida. Os pacientes haviam recebido tratamento clínico medicamentoso por pelo menos dois meses, sem sucesso. Após um mês de seguimento, houve redução de $37,4 \%$ na espessura foveal, que se manteve em três e seis meses. A acuidade visual melhorou em 8 (80\%) olhos. Em três e seis meses, 30\% dos olhos mantiveram-se estáveis e o restante manteve a melhor acuidade visual em comparação ao início do estudo. Este estudo sugere que a infiltração intra-ocular com triamcinolona pode ser eficaz em casos de edema macular cistóide associado à doença de Behçet.

Tunc R, Saip S, Siva A, Yazici H: Cerebral venous thrombosis is associated with major vessel disease in Behçet's syndrome (Trombose cerebral é associada com doença de vasos maiores em síndrome de Behçet). Ann Rheum Dis 63: 1693 4, 2004. Behçet's Syndrome Research Centre, Department of Internal Medicine, Division of Rheumatology, Cerrahpasa School of Medicine, Istambul, Turquia.

Foram estudados 88 pacientes com doença de Behçet com envolvimento do sistema nervoso central e 80 pacientes com doença de Behçet sem comprometimento neurológico, como grupo controle. A freqüência de envolvimento de vasos de maior calibre foi $29 \%$ no grupo com doença do sistema nervoso central e $23 \%$ no grupo controle $(P=0,374)$. A análise foi estratificada pelo tipo de doença neurológica: 7/11 (64\%) pacientes com trombose de seio dural tiveram comprometimento de vaso de maior calibre (trombose de veias maiores em todos os pacientes, em um deles com envolvimento pulmonar e em outro com comprometimento da artéria ilíaca), em comparação à 15/77 (19\%) pacientes com envolvimento inflamatório parenquimatoso do encéfalo $(P=0,004)$. A idade média dos pacientes com trombose de seio dural foi menor que a idade média dos pacientes com inflamação parenquimatosa $(23,1 \pm 8,8$ vs $32,0 \pm 7,5$ anos; $P=0,002)$. Este estudo sugere um mecanismo patogênico comum para envolvimento vascular sistêmico e no sistema nervoso central, reforçando a idéia do subtipo de pacientes com "doença de Behçet vascular”, com maior risco de vasculite de vasos de maior calibre e/ou eventos trombóticos.

Leiba M, Seligsohn U, Sidi Y, et al: Thrombophilic factors are not the leading cause of thrombosis in Behçet's disease (Fatores trombofílicos não são a causa predominante de trombose em doença de Behçet). Ann Rheum Dis 63: 1445-9, 2004. Instituição: Department of Medicine C, Sheba Medical Centre, TelHashomer, Israel.

Estudos sobre a associação de trombose e trombofilia em pacientes com doença de Behçet têm apresentado resultados controversos. Neste contexto, os pacientes avaliaram amostras de sangue de 107 pacientes com e sem trombose para a frequiência de Fator V de Leyden, polimorfismo G20210A do gene protrombina, polimorfismo C 677T do gene metiltetrahidrofolato redutase, nível de Fator VIII, concentrações de homocisteína e proteína C reativa, dislipidemia e glucosilceramida plasmática. Os pacientes com trombose $(\mathrm{n}=33)$ apresentaram maior prevalência de úlceras genitais, doença ocular, envolvimento gastrintestinal e do sistema nervoso central. Os pacientes com trombose apresentaram níveis mais elevados de Fator VIII, colesterol total, VLDL e apoliproteínas B-100, C-II e C-III, mas não existiu diferença significativa nos fatores trombofílicos estudados. Em análise multivariada, manteve-se a associação entre hipertrigliceridemia e trombose [OR CI95\% 1,58 (1,09-2,30), para uma diferença de $40 \mathrm{mg} / \mathrm{L}]$. Os autores concluem que trombofilia não parece ser um determinante da existência de trombose em pacientes com doença de Behçet, mas dislipidemia, principalmente hipertrigliceridemia, podem ser fatores de risco. 
Stanford M, Whittall T, Bergmeier LA, et al: Oral tolerization with peptide 336-351 linked to cholera toxin B subunit in preventing relapses of uveitis in Behçet's disease (Desensibilização com péptide 336-351 associado à subunidade B da toxina colérica em prevenção de recidivas de uveíte em pacientes com doença de Behçet). Clin Exp Immunol 137: 201 8, 2004. Department of Ophthalmology, Guy's, King's and St. Thomas' School of Medicine and Dentistry, Guy's Hospital, Londres, Inglaterra.

O peptídio específico da doença de Behçet (336-351) foi identificado na proteína de choque térmico heat shock $60 \mathrm{Kd}$ (HSP60). P331-351 oral induziu uveíte em ratos, prevenida pela desensibilização com o peptídio ligado à subunidade B de toxina colérica recombinante (CTB). Foi realizado um estudo de fase I/II onde oito pacientes com doença de Behçet receberam 331-351-CTB oral, 3 vezes por semana, seguido por retirada progressiva dos imunossupressores. Não houve efeitos adversos e a retirada dos imunossupressores não causou recidiva em $5 / 8$ pacientes. Após o tratamento de desensibilização, $3 / 5$ dos pacientes mantiveram-se sem atividade inflamatória ocular por 10-
18 meses após a cessação de todos os tratamentos. O controle da uveíte e das manifestações extra-oculares associou-se à ausência de proliferação de células $\mathrm{CD} 4+$, a uma redução na expressão de células TH1 (CCR5 e CXCR3), menor expressão de INF-gama e TNF, células CCR7+ e moléculas co-estimuladoras (CD40 e CD28), em comparação com o aumento destes parâmetros nos pacientes com recidiva da uveíte. A eficácia da terapia de desensibilização oral com peptídio 331-351-CTB deve ser avaliada em estudos de fase III, mas esta nova estratégia pode ser útil em pacientes com doença de Behçet ou outras doenças auto-imunes em que se identificarem peptídio específicos.

Tomonari A, Tojo A, Takahashi T, et al: Resolution of Behçet's disease after HLA-mismatched unrelated cord blood transplantation for myelodysplastic syndrome (Resolução de doença de Behçet após transplante de células tronco de cordão de doador não relacionado, HLA-compatível para síndrome mielodisplásica). Ann Hematol. 83: 464-6, 2004. Instituição: Department of Hematology/Oncology, The Institute of Medical Science, The University of Tokyo, Japão.

Neste trabalho, os autores descrevem o caso de uma jovem que desde 1991 apresentava doença de Behçet manifesta por aftose bipolar, febre e eritema nodoso. Sua doença vinha se mostrando refratária ao tratamento usual. Em 1999, confirmou-se o diagnóstico de síndrome mielodisplásica (anemia refratária com excesso de blastos). Como a neutropenia da paciente piorava (e os sintomas da doença de Behçet se mantinham), a paciente foi submetida em junho de 2001 a transplante de células-tronco de cordão de doador não relacionado. Houve doença enxerto-versuso-hospedeiro grau I e a ciclosporina foi progressivamente reduzida até sua suspensão seis meses após o procedimento. Após um período de observação de 23 meses, a paciente mantém-se assintomática, tanto das alterações da mielodisplasia quanto dos sintomas da doença de Behçet. Estes dados sugerem que transplante de células-tronco pode ser indicado em pacientes com doença de Behçet grave e refratária ao tratamento usual.

Kotter I, Gunaydin I, Zierhut M, Stubiger N: The use of interferon alpha in Behçet's disease: review of the literature (O uso de interferon alfa em doença de Behçet: revisão da literatura). Semin Arthritis Rheum 33: 320-35, 2004. Instituição: University Hospital, Department of Internal Medicine II, Tubingen, Alemanha.

Os autores realizaram revisão da literatura sobre o uso de interferon alfa em doença de Behçet, através do banco de dados PubMed e anais de congressos. Foram incluídos na análise 34 artigos originais e quatro resumos de congressos, com um total de 338 pacientes, sendo 182 com doença inflamatória ocular aguda. Duzentos e sessenta e quatro pacientes receberam interferon alfa- 2 a e 74 interferon alfa-2b. O índice de resposta parcial ou completa variou de 86\% para sintomas muco-cutâneos, 96\% para artrite e $94 \%$ para uveíte. Doses mais altas foram associadas 
à melhor resposta, com até $56 \%$ de remissão sustentada após cessação do tratamento, mas com maior índice de efeitos adversos, como acontece em pacientes com hepatite C. Houve melhor resposta com interferon alfa-2a, mas este resultado pode ser conseqüência de viés pelo maior número de casos estudados com esta formulação. A comparação dos trabalhos foi prejudicada por metodologias diferentes, mas os autores concluem que interferon alfa é eficaz nas diferentes manifestações clínicas inflamatórias da doença de Behçet.

Siva A, Altintas A, Saip S: Behçet's syndrome and the nervous system (Síndrome de Behçet e o sistema nervoso central.) Curr Opin Neurol 17: 347-357, 2004. Instituição: Department of Neurology, Clinical Neuroimmunology Division, Cerrahpasa School of Medicine, University of Istanbul, Cerrahpasa, Istambul, Turquia.

Neste artigo de revisão, os autores discutem os principais aspectos do envolvimento no sistema nervoso central na doença de Behçet. Tal comprometimento acontece de 5\% a $10 \%$ dos pacientes, é mais freqüente em homens e pode ser classificado em duas formas principais: a primeira é usualmente atribuída à inflamação de vasos de menor calibre e apresenta-se como lesões parenquimatosas focais ou multifocais, denominada comprometimento intra-axial; a outra forma de acometimento do sistema nervoso central seriam as tromboses de seios venosos durais e hipertensão intracraniana, menos freqüentes, com melhor prognóstico e descritas como extra-axial. As duas formas podem eventualmente coincidir em um mesmo paciente e outras manifestações menos freqüentes, como quadros psiquiátricos ou neuropatia periférica, também são descritas. $\mathrm{Na}$ experiência dos autores, uma proporção significativa dos pacientes apresenta cefaléia sem substrato fisiopatológico definido, entendida como uma manifestação inespecífica da doença. Sintomas neurológicos podem ser ainda decorrentes do tratamento, como a neurotoxicidade da ciclosporina, conseqüência de outras manifestações da doença, como trombo-embolismo a partir de envolvimento cardíaco da doença de Behçet ou complicações infecciosas, especialmente em pacientes já em uso de corticóide e/ou imunossupressores. O espectro de apresentação clínica inclui envolvimento do trato córtico-espinhal com ou sem sintomas cerebelares, neuropatias cranianas, confusão mental ou outras síndromes neuro-comportamentais. O curso clínico pode se apresentar como um episódio isolado e com recuperação, com ou sem seqüelas, episódios recorrentes com remissão intermitente e progressão secundária ou, em menor freqüência, um curso primariamente progressivo. Em sua forma extra-axial (trombose de seio dural) a doença pode se apresentar como episódio isolado. Ainda que do ponto de vista neurológico tenha melhor prognóstico que a forma intra-axial, pode se associar ao comprometimento extra-craniano de vasos de maior calibre, potencialmente grave. Métodos de imagem, como a ressonância nuclear magnética, são a primeira linha na avaliação propedêutica, complementada por avaliação do líquor e estudos neurofisiológicos. O tratamento segue as diretrizes gerais da doença com uso de corticóide, associado ou não a imunossupressores, nas formas inflamatórias e anticoagulação, associada ou não a corticóide, na forma extraaxial. O envolvimento do sistema nervoso central é um marcador importante de prognóstico, com morbi-mortalidade significativa elevada (até 50\%) em períodos de observação de até dez anos.

A doença de Behçet mantém-se como uma condição clínica associada a complicações graves, com morbi-mortalidade expressiva. No entanto, ainda que existam perguntas a serem respondidas, ocorre um progresso evidente no entendimento de seus processos fisiopatológicos, na caracterização de seu curso clínico e especificamente na documentação de estratégias de tratamento mais eficazes. O médico reumatologista, conhecedor destes aspectos, tem a oportunidade do diagnóstico correto da doença e suas conseqüências, definição do prognóstico nas suas diferentes formas de apresentação e intervenção terapêutica ajustada, logrando, na maior parte dos casos, modificação do curso natural da doença e melhoria da qualidade de vida dos pacientes. 